\title{
Projeto Atenção à Mãe e à Adolescente: Alcances e Desafios
}

\author{
George Wallisson Severo de Sá $a^{1}$; José Carlos Apolonio da Silva² ; Juliana Silveira de Melo ${ }^{3}$; Ana Olívia de Oliveira \\ Marinho ${ }^{4}$; Gislene Farias de Oliveira ${ }^{5}$
}

\begin{abstract}
Resumo: Seu entendimento é amplo, porém, vulnerabilidade refere-se a uma predisposição que cada indivíduo possui para apresentar resultados negativos no desenvolvimento. Entre os diversos fatores protetores que potencializam os riscos inerentes em um ambiente vulnerável, destaca-se a educação, a qual é considerada principal instrumento para a elevação dos níveis de capital humano e para promover o bem-estar de jovens e adolescentes. É nesse sentido que a extensão universitária se encaixa na comunidade na qual está inserida, fazendo uma ponte para que os conhecimentos em saúde possam chegar até a sociedade circunvizinha. Objetiva-se com este estudo caracterizar o perfil da população assistida pelo Projeto Atenção à Mãe e à Adolescente (AMA) no tocante à vulnerabilidade social e econômica na qual se inserem. Realizou-se uma pesquisa bibliográfica e de campo, na qual se utilizou o método indutivo e estatístico, embasando-se na técnica qualitativo-descritiva em observação direta da problemática vulnerabilidade. Lançando mão de um questionário para o levantamento dos dados foi possível conhecer as condições relativas à vulnerabilidade social e econômica da comunidade estudada. A amostra foi composta por 66 participantes das mais variadas faixas etárias, que frequentaram as atividades do projeto AMA durante o ano de 2012. Os dados mostram que a maioria desse público presencia frequentemente pessoas em situações deploráveis, e direta ou indiretamente acabam se expondo a fatores de exposição como prostituição, uso de drogas lícitas e ilícitas e violência familiar. A ação de extensão AMA com sua metodologia de execução constituise um importante fator protetivo para esse público, sendo bastante salutar para amortizar a situação de risco que essa população vive a partir de um processo educativo em saúde.
\end{abstract}

Palavras-chave: mãe adolescente, desenvolvimento, gravidez

\section{Project Attention to the Mother and Adolescent: Scope and Challenges}

\begin{abstract}
Its understanding is wide, however, vulnerability refers to a predisposition that each individual has to display negative development results. Among the many protective factors that enhance the risks inherent in a vulnerable environmentstands out education, which is considered the main instrument for the higher levels of human capital and promote the wellbeing of children and young people. It is in this sense that the university extension fits into the community in which is inserted, making a bridge for the health knowledge can reach the surrounding society. Objectively with this study to characterize the profile of the population assisted by the Project Attetion for the Mother and Adolescent (AMA) concerning social and economic vulnerability in which they are inserted. We conducted a bibliographic and field research, in which we used the inductive and statistical method, basing on the qualitative-descriptive technique in direct observation of problematic vulnerability. By making use of a questionnaire for the survey of the data it was possible to meet the conditions for the social and economic vulnerability of the community studied. The sample consisted of 66 participants from various age groups who attended the activities of AMA project during the year 2012. The data show that the majority of this public often witnesses people in deplorable situations, and directly or indirectly end up exposing the exposure factors such as prostitution, use of licit and illicit drugs and family violence. The action of AMA extension with its implementation methodology constitutes an important protective factor for this public, being very beneficial to amortize the risk that this population lives from a health education process.
\end{abstract}

Keywords: teen mom, development, pregnancy

\footnotetext{
${ }^{1}$ Acadêmico de Medicina da Universidade Federal do Ceará - Campus de Barbalha. E-mail: George_jua@hotmail.com;

2 Acadêmico de Medicina da Universidade Federal do Ceará - Campus de Barbalha. E-mail: zeapolonio@hotmail.com;

${ }_{3}^{3}$ Acadêmico de Medicina da Universidade Federal do Ceará - Campus de Barbalha.

${ }_{3}^{3}$ Acadêmica de Medicina da Universidade de Pernambuco - UPE, Pernambuco, Brasil. E-mail: fantinhapx@gmail.com;

${ }^{3}$ Professora da Universidade Federal do Ceará - Campus de Barbalha. E-mail: gislenefarias@ gmail.com
} 


\section{Introdução}

Os indicadores sociais do Brasil vêm melhorando ao longo dos anos. Para se ter uma ideia, a taxa de mortalidade infantil no Brasil caiu 29,0\% no período de 1999 a 2009. Ao contrário, a expectativa de vida vem crescendo e situa-se em torno de 72,9 anos ao nascer, além disso, os indicadores sobre saúde e educação são positivos (ROGERS e BALLANTYNE, 2008). Entretanto, esse fenômeno de prosperidade não se estende a todas as comunidades brasileiras e muitas delas ainda amargam grandes adversidades e por isso se encontram em situação de vulnerabilidade. Seu entendimento é amplo, porém, vulnerabilidade refere-se a uma predisposição que cada indivíduo possui para apresentar resultados negativos no desenvolvimento, existindo como uma possibilidade, não como uma certeza (INSTITUTO BRASILEIRO DE GEOGRAFIA E ESTATÍSTICA , 2010; CECCONELLO, 2003). Nesse contexto, ambientes vulneráveis são, portanto, aqueles que oferecem riscos para os indivíduos, com a impossibilidade de acesso e condições dignas habitacionais, sanitárias, educacionais e de trabalho, fato que lhes restringem o acesso às oportunidades (PERONA, 2012; MELO, 2011).

As fontes de vulnerabilidade podem ser distintas como extrínseca ocasionada por circunstâncias externas ao indivíduo, como falta de poder socioeconômico, falta de escolaridade ou carência de recursos; ou intrínseca causada por características inerentes ao próprio indivíduo, tais como doença mental, deficiência intelectual, ou extremos de idade (crianças e idosos) (ROGERS e BALLANTYNE, 2008). Portanto, o indivíduo pode estar vulnerável em várias esferas, seja ela política, econômica ou social (CECCONELLO, 2003).

A prevalência de fatores de risco na adolescência e na juventude, por ser um período da vida caracterizado por mudanças físicas e psicológicas, além de ser marcado por descobertas de várias situações com as quais nem sempre sabe lidar adequadamente, nos impõe uma atenção maior a esse público (AMPARO, 2008; SAPIENZA e PEDROMÔNICO , 2012). É desanimador para o jovem, por exemplo, que empreende sua primeira inserção no mercado de trabalho, pois, paradoxalmente, boa parte dos contratantes exige experiência profissional para quem busca o primeiro emprego, além disso, a desqualificação profissional os impõe baixos salários. Outro fator preocupante é a exposição à violência e ao uso de drogas lícitas e ilícitas, pois os índices de pessoas vulneráveis são particularmente altos nessa faixa etária e, de uma maneira geral, leva-os a morte precoce (CASTRO e ABRAMOVAY, 2002).

Apesar de não poder ser analisado isoladamente no contexto social da vulnerabilidade, o rendimento familiar ainda nos permite avaliar sobremaneira o grau de risco de uma população ou indivíduo. A pobreza constitui risco potencial ao desenvolvimento das pessoas, muitas vezes limitando 
o acesso delas a oportunidade de ascensão social, financeira ou intelectual. Além disso, o risco pobreza tende a afetar a relação conjugal, desarticulando famílias, e oferecendo riscos a saúde. Por se constituir um espaço tradicional de socialização para o jovem, a família é muito importante para ele e quando o lar deixa de ser um espaço de proteção para ser um espaço de conflito, a superação desta situação é deveras dificultosa para uma família que vive na miséria, pois essa família não dispõe de redes de ajuda para a superação das adversidades, resultando assim, na sua desestruturação (CECCONELLO, MELO, FONSECA e LIMA, 2011; 2003; GOMES, 2003; ABRAMOVAY, 2002).

No combate aos fatores de risco que exacerbam a vulnerabilidade, posicionam-se contra eles os fatores de proteção, pois aumentam as vivências resilientes e exiguificam os agentes potencialmente danosos ao desenvolvimento do indivíduo (AMPARO, 2008; AMPARO, 2012). Dentre os fatores de proteção, destacam-se: 1. Características individuais como autoestima, inteligência, competência social e autocontrole. 2. Fatores familiares como coesão, vínculo positivo, estabilidade e suporte. 3 . Fatores relacionados à relação positiva do meio como apoio social de igrejas, grupos de amigos e professores (CECCONELLO , 2003; AMPARO, 2008).

Ainda em se tratando dos fatores de proteção, que tendem a atenuar os efeitos negativos dos riscos ${ }^{6}$, destaca-se a educação. Sua importância como fator protetor é primordial, pois é considerado o principal instrumento para a elevação dos níveis de capital humano e para promover o bem-estar de jovens e adolescentes, além de ser otimizador das potencialidades do indivíduo, proporcionando-lhe a interação interpessoal e a sua atuação como cidadão ativo em comunidade, além de favorecer sua ascensão social e pessoal (ABRAMOVAY,, 2002; SILVA, 2007).

Nesse contexto, a educação torna-se fator protetor da vida e auxiliador da saúde na construção e transição do conhecimento para população e desde as campanhas higienistas no século XIX, a educação em saúde vem tomando força no Brasil. É nesse sentido que a extensão universitária se encaixa na comunidade na qual está inserida, fazendo uma ponte para que os conhecimentos em saúde possam chegar até a sociedade circunvizinha, permitindo a troca de conhecimentos científicos e populares, mas principalmente, atuando na redução da vulnerabilidade da comunidade desassistida. Portanto, a extensão universitária é um instrumento potencializador de mudanças (ACIOLI , 2008).

Objetiva-se com este estudo caracterizar o perfil da população assistida pelo Projeto Atenção à Mãe e à Adolescente (AMA) no tocante à vulnerabilidade social e econômica na qual se inserem, além da aceitação e contribuição do projeto AMA para a comunidade. 


\section{Método}

Realizou-se uma pesquisa bibliográfica e de campo, na qual se utilizou o método indutivo e estatístico, embasando-se na técnica qualitativo-descritiva em observação direta da problemática vulnerabilidade, além disso, lançando mão de um questionário para o levantamento dos dados foi possível conhecer as condições relativas à vulnerabilidade social e econômica da comunidade estudada, além de identificar fatores de risco os quais assolam a população que faz parte presente projeto. O mesmo vem sendo desenvolvido com o apoio Associação Maria Mãe da Vida, uma Organização filantrópica e católica que presta um trabalho assistencialista em diversas cidades no Nordeste.

Os critérios de inclusão da presente pesquisa foram: 1. Estar devidamente matriculada na Associação Maria Mãe da Vida e, 2. Ter participado de pelo menos três encontros do projeto AMA.

\section{Resultados}

A amostra foi composta por 66 participantes das mais variadas faixas etárias, desde os dez a mais de sessenta anos de idade, que frequentaram as atividades do projeto AMA durante o ano de 2012.

No tocante ao gênero observou-se preponderância do sexo feminino, perfazendo $76 \%$ do total de pesquisados, a maioria composta por adolescentes de dez a dezessete anos $(66,7 \%)$. No quesito ocupação, 66,7\% da amostra alegava apenas estudar, sendo que frequentavam as escolas públicas de seu bairro. Além disso, devido ao público de faixa etária mais baixa, é compreensível o achado de $60,6 \%$ desse público possuir apenas o ensino fundamental incompleto.

Tabela 1: Caracterização da amostra de acordo com a faixa etária e ocupação.

\begin{tabular}{|lll|lll|}
\hline Faixa Etária & $\mathbf{N}$ & $\%$ & Ocupação & $\mathbf{N}$ & $\%$ \\
\hline $\mathbf{1 0 - 1 7}$ anos & 44 & 66,7 & Estudante & 44 & 66,7 \\
\hline $\mathbf{1 8 - 2 4}$ anos & 04 & 6,0 & Doméstica & 14 & 21,2 \\
\hline $\mathbf{2 5 - 6 0}$ anos & 16 & 24,3 & Aposentada & 02 & 3,1 \\
\hline Mais de 60 anos & 02 & 3,0 & Do lar & 05 & 7,5 \\
\hline & & Sem ocupação & 01 & 1,5 \\
\hline
\end{tabular}

$\mathrm{N}$ : número absoluto de pessoas.

Fonte: Dados da pesquisa (2012). 
Como é perceptível pela análise da tabela 1, a maioria da amostra é composta de adolescentes. Sanchez et al. e Amparo et al. mostraram em seus estudos que nessa fase do desenvolvimento as decisões do indivíduo são sobremaneira influenciáveis tornando esses jovens, portanto, mais facilmente ludibriáveis e aliciáveis, além de ser uma fase de descobertas, da busca pelo prazer, por novas sensações. Por isso, a adolescência por si só já é considerada um fator de risco (IBGE, 2010; AMPARO, 2008; SANCHEZ, et al, 2010).

Ainda de acordo com a tabela 1 percebemos o alto número de estudantes que frequentam o projeto, isso seria muito positivo tendo em vista o quanto a educação é um importante fator de proteção (AMPARO, 2008). Entretanto, todos esses estudantes frequentam escolas públicas da região que, é sabido, tendem a uma baixa qualidade de ensino, quando comparadas às escolas particulares. Portanto, ao invés de ser um fator de proteção, a escola não consegue fornecer o suporte adequado para esses estudantes progredirem academicamente e profissionalmente, assim dificultando para eles alcançarem níveis sociais e econômicos mais confortáveis, favorecendo uma melhor qualidade de vida (ABRAMOVAY et al, 2002).

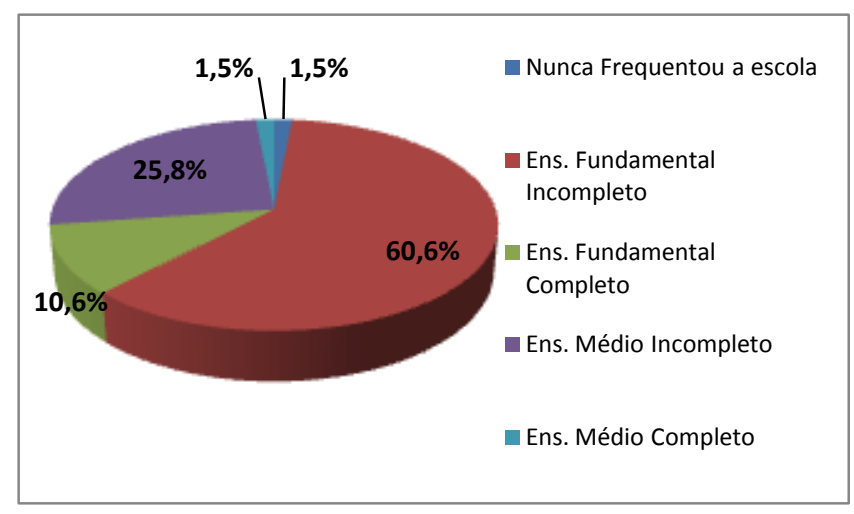

Gráfico 1: Caracterização da amostra de acordo com a escolaridade

Fonte: Dados da pesquisa (2012). 


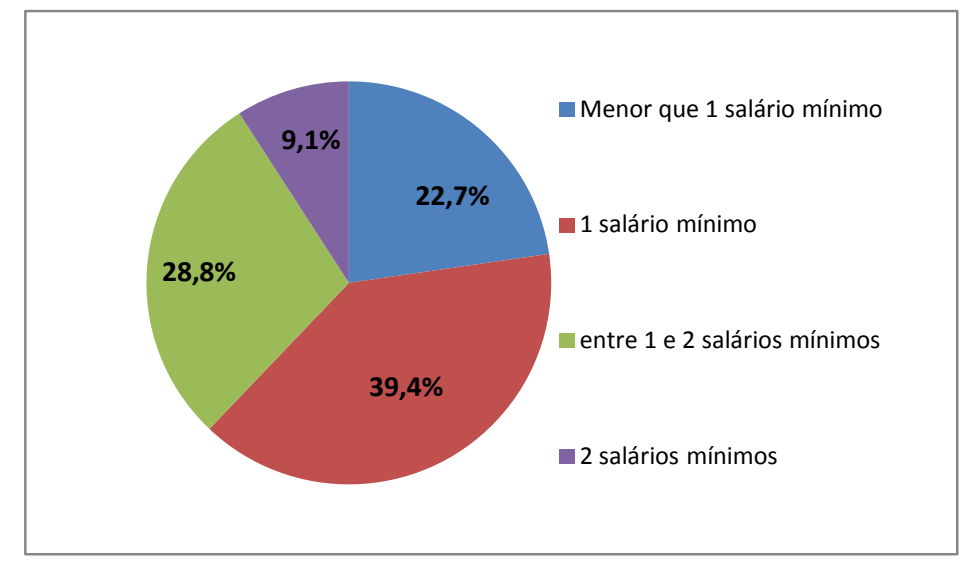

Gráfico 2: Caracterização da amostra de acordo com a renda

Fonte: Dados da pesquisa (2012).

A partir da análise do gráfico 1 é possível depreender o quanto a população estudada tem uma baixa escolaridade, apesar de 57,6\% relatar que a educação a qual receberam é de boa qualidade, constituindo-se mais um fator de risco para esse público.

O fato da maior parte desse público possuir apenas o ensino fundamental incompleto $(60,6 \%)$ pode ser decorrente da grande quantidade de adolescentes da faixa etária de 10 a 17 anos (66,7\%) que frequentam o projeto, entretanto, mesmo esse fato sendo verdade, denota-se a partir desse entendimento que pelo menos $33,3 \%$ dos pesquisados possui mais de 18 anos de idade e apenas 1,5\% da amostra possui ensino médio completo. Público esse que já deveria ter concluído o ensino médio.

Apenas $1,5 \%$ deles alcançaram esse patamar acadêmico, corroborando com o entendimento de que se tem um grande número de pessoas com grau de escolaridade baixo. Situação tendenciosa que aumenta a vulnerabilidade desses jovens, pois, em geral, maiores níveis de escolaridade garantem melhores oportunidades de inserção qualificada no mercado de trabalho que lhes proporcionariam favorável qualidade de vida (IBGE, 2010; ABRAMOVAY, et al, 2002).

Em face disso, esse baixo estágio de classificação profissional justifica a renda cuja amostra demonstrou que em sua maioria, 90,9\%, é menor do que dois salários mínimos por mês. Essa situação econômica se agrava quando constatamos na pesquisa o percentual de 54,5\% desse público residindo com 5 a 7 pessoas em suas casas. Neste caso, reduzindo a renda per capta mensal. Essa situação financeira torna extremamente vulnerável o público em estudo, pois a pobreza pode colocar em xeque o desenvolvimento intelectual, a salubridade da moradia, as condições de saúde, enfim, a pobreza tange negativamente vários aspectos dessas famílias (CECCONELLO, 2003; HUTZ e SILVA, 2002). 
A ocupação, como retratado na tabela 1, reflete o nível de escolaridade desse público, tendo em vista que a única atividade laboral relatada foi doméstica, perfazendo $21,2 \%$, profissão que não demanda elevado grau de estudo ou de qualificação, entretanto remunera mal.

A despeito da situação econômica na qual se encontram, 60,6\% relata ser suficiente a renda que possuem, refletindo como são limitadas as aspirações e expectativas desse público em face da situação econômica e social na qual vivem. Muitas vezes deixem de qualificar-se para o mercado de trabalho. Outra situação de vulnerabilidade é a saúde, tendo em vista que 98,5\% da amostra diz tế acesso ao serviço de saúde, porém apenas 21,2\% referem ser esse serviço prestado de maneira adequada, de qualidade, de forma a atender suas necessidades.

Tabela 2: Caracterização dos fatores de risco para a população.

\begin{tabular}{|c|c|c|}
\hline Fatores de Risco & SIM & NÃO \\
\hline Contato com prostituição & $33,3 \%$ & $66,7 \%$ \\
\hline $\begin{array}{l}\text { Contato com usuários de drogas } \\
\text { ilícitas }\end{array}$ & $81,9 \%$ & $18,1 \%$ \\
\hline $\begin{array}{l}\text { Contato com o tráfico de drogas } \\
\text { ilícitas }\end{array}$ & $57,6 \%$ & $42,4 \%$ \\
\hline Contato com o alcoolismo & $95,5 \%$ & $4,5 \%$ \\
\hline Contato com a violência familiar & $63,6 \%$ & $36,4 \%$ \\
\hline
\end{tabular}

Fonte: Dados da pesquisa (2012)

A tabela 2 evidencia mais cinco fatores de risco aos quais a população em estudo encontra-se exposta. Os dados mostram que a maioria desse público presencia frequentemente pessoas em situações deploráveis, e direta ou indiretamente acabam se expondo a fatores de exposição como prostituição, uso de drogas lícitas e ilícitas e violência familiar. As drogas, em particular para esse contexto, em que temos um grande número de adolescentes, é uma fator de risco muito importante, pois é uma fase de maior vulnerabilidade para o ingresso no uso dessas substâncias, além do fato de a simples disponibilidade da droga já ser considerado um fator de predisposição ao seu uso (IBGE, 2010; SANCHEZ, 2010; PATTON et al, 2004). Os riscos inerentes à dinâmica familiar também são evidenciados na tabela 2, a violência familiar e o alcoolismo estão presentes na vida das pessoas pesquisadas, este último e destacando com 95,5\%. Esses dois últimos quesitos são importantes fatores de desarticulação familiar que acaba pondo em xeque o importante fator de proteção família (MELO, 
FONSECA e LIMA, 2011; ABRAMOVAY, 2002; AMPARO et al, 2008; HANADA, D'OLIVEIRA e SCHRAIBER, 2010).

Nesse contexto de vulnerabilidades o projeto de extensão Atenção à Mãe e à Adolescente (AMA), alicerçando suas atividades na prevenção dos agravos à saúde a partir da metodologia educacional, na transmissão de informações, sendo esse um importante fator protetivo (SANCHEZ et al, 2012). É fundamental na amortização dos impactos que os fatores de risco, observados na pesquisa, outorgam ao público assistido pelo projeto, principalmente porque $62,1 \%$ dos participantes relataram que em sua formação acadêmica não tiveram abordados os assuntos contemplados pelo projeto AMA. Além disso, a forma lúdica e dinâmica cuja informação é transmitida fornece uma boa aceitação ao projeto e ao tema contemplado já que 89,4\% alegam apreciar a didática e as informações do projeto. É importante frisar o impacto dessas informações na vida prática dos participantes já que 98,5\% dos questionados afirmaram o quanto os assuntos abordados pelo projeto são importantes na sua vida diária e aplicáveis em seu cotidiano.

\section{Conclusão}

Devido à inserção do público assistido pelo projeto AMA em uma situação de pobreza, de carência intelectual e econômica, de convívio com drogas lícitas e ilícitas, de prostituição e de desarranjo familiar, enfim, de inúmeros fatores de agravo social que colocam a vida dessas pessoas em risco, a ação de extensão AMA com sua metodologia de execução e, abordagem de temas do cotidiano constitui-se um importante fator protetivo para esse público, sendo bastante salutar para amortizar a situação de risco que essa população vive a partir de um processo educativo em saúde, tendo em vista que, comprovadamente, a educação constitui-se em um fator de proteção.

\section{Referências}

ABRAMOVAY, Miriam et al. Juventude, violência e vulnerabilidade social na América Latina: desafios para políticas públicas. Brasília: UNESCO, 2002. 92 p.

ACIOLI, Sonia. A prática educativa como expressão do cuidado em Saúde Pública. Revista Brasileira de Enfermagem. Brasília: Associação Brasileira de Enfermagem. v.61, n.1, p.117-121, jan-fev 2008.

Id en line Revista de Psicologia. Ano 8, No. 22, Fevereiro/2014 - ISSN 1981-1179. Edição eletrônica em http://idonline.emnuvens.com.br/id 
AMPARO, Deise Matos do et al. A escola e as perspectivas educacionais de jovens em situação de risco. Psicologia Escolar e Educacional. Campinas: ABRAPEE. n.1, v.12, p.69-88, jan-fev 2008.

AMPARO, Deise Matos do et al. Adolescentes e jovens em situação de risco psicossocial: redes de apoio social e fatores pessoais de proteção. Estudos de Psicologia. Natal: PPgPsi-UFRN. v.13, n.2, p.165-174, 2008. Disponível em: <http://www.scielo.br/scielo.php?script=sci_arttext\&pid=S1413294X2008000200009\&lng=pt\&nrm=iso>. Acesso em: 15 jul. 2012.

CASTRO, Mary Garcia; ABRAMOVAY, Miriam. Jovens em situação de pobreza, vulnerabilidades sociais e violências. Cadernos de Pesquisa. São Paulo: Fundação Carlos Chagas. n.116, p.143-176, jul. 2002.

CECCONELLO, Alessandra Marques. Resiliência e vulnerabilidade em famílias em situação de risco. 2003. 320f. Tese (Doutorado em Psicologia) - Universidade Federal do Rio Grande do Sul, Porto Alegre.

GOMES, Mônica Araújo. Filhos de ninguém? Um estudo das representações sociais sobre família de adolescentes em situação de rua. 2003. 124f. Dissertação (Mestrado Profissional em Saúde da Criança e do Adolescente) - Universidade Estadual do Ceará, Fortaleza, 24 fev. 2003.

HANADA, Heloisa; D’OLIVEIRA, Ana Flávia Pires Lucas; SCHRAIBER, Lilia Blima. Os psicólogos na rede de assistência a mulheres em situação de violência. Revista Estudos Feministas. Florianópolis: UFSC. v.18, n.1, p.33-59, jan.-abr. 2010.

HUTZ, Claudio Simon; SILVA, Débora Frizzo Macagnan. Avaliação Psicológica com crianças 5 adolescentes em situação de risco. Avaliação Psicológica. Itatiba: IBAP. v.1, n.1, p.73-79, 2002.

INSTITUTO BRASILEIRO DE GEOGRAFIA E ESTATÍSTICA. Síntese de indicadores sociais: uma análise das condições de vida da população brasileira. Rio de Janeiro: IBGE, 2010. 317 p.

MARCONI, Marina de Andrade; LAKATOS, Eva Maria. Fundamentos da metodologia científica. 6. ed. São Paulo: Atlas, 2009. 315 p.

MELO, Marlete Euná Brito de; FONSECA, Evanilda de Brito; LIMA, Leitisomeire Brito de. A criança em um contexto de vulnerabilidade social. In: COLÓQUIO INTERNACIONAL: EDUCAÇÃO E CONTEMPORANEIDADE. 5, 2011, Sergipe. 
PATTON, George et al. Puberty and the Onset of Substance Use and Abuse. Pediatrics. Elk Grove Village: American Academy of Pediatrics v.114, n. 3, 1 set. 2004, p.300-306.

PERONA, Nélida et al. Vulnerabilidad y exclusión social: uma propuesta metodológica para el estúdio de las condiciones de vida de los hogares. Kairos. San Luís: UNSL. N.8, jan.-fev. 2001.

ROGERS, Wendy; BALLANTYNE, Angela. Populações especiais: vulnerabilidade e proteção. Revista Eletrônica de Comunicação, Informação \& Inovação em Saúde. Rio de Janeiro: Fundação Carlos Chagas. v.2, sup.1, p31-41, dez. 2008.

SANCHEZ, Zila van der Meer et al. Papel da informação como medida preventiva ao uso de drogas entre jovens em situação de risco. Ciência \& Saúde Coletiva. Rio de Janeiro: ABRASCO. v.15, n.3, p.699-708, 2010..

SAPIENZA, Graziela; PEDROMÔNICO, Márcia Regina Marcondes. Risco, proteção e resiliência no desenvolvimento da criança e do adolescente. Psicologia em Estudo. Maringá: Universidade Estadual de Maringá v.10, n.2, p.209-216, maio-ago. 2005.

SILVA, Algéria Varela da. Vulnerabilidade social e suas consequências: o contexto educacional da juventude na Região Metropolitana de Natal. In: ENCONTRO DE CIÊNCIAS SOCIAIS DO NORTE NORDESTE. 13, 2007, Maceió.

\section{Como citar este artigo (Formato ISO):}

SÁ, G.W.S.; SILVA, J.C.A.; MELO, J.S.; MARINHO, A.O.O.; OLIVEIRA, G.F. Projeto Atenção à mãe e à adolescente: alcances e desafios. Id on Line Revista de Psicologia, Fevereiro de 2014, vol.8, n.22, p. 216-225. ISSN 1981-1189.

Recebido: 29/11/2013

Aceito: 03/02/2014 